\title{
Asymptotic Regularity and Uniform Attractor for Non-autonomous Viscoelastic Equations with Memory
}

\author{
Ye Zeng*,Yanan Li, Yongqin Xie and Shuangli Luo \\ School of Mathematics and Statistics, Changsha University of Science and Technology, Changsha, China
}

\begin{abstract}
In this paper, long-time behavior of a class of non-autonomous viscoelastic equations with fading memory is investigated. We establish the existence of a compact uniform attractor together with its structure in $H_{0}^{1}(\Omega) \times H_{0}^{1}(\Omega) \times L_{\mu}^{2}\left(R^{+} ; H_{0}^{1}(\Omega)\right)$. The compact uniform
\end{abstract} attractor is bounded in $D(A) \times D(A) \times L_{\mu}^{2}\left(R^{+} ; D(A)\right)$ and attracts every bounded set of $H_{0}^{1}(\Omega) \times H_{0}^{1}(\Omega) \times L_{\mu}^{2}\left(R^{+} ; H_{0}^{1}(\Omega)\right.$ ).

Keywords-non-autonomous wave equations; regularity; uniform attractor; memory;viscoelasticity

asymptotic

\section{INTRODUCTION}

In this paper, we consider the dynamical behavior of the solutions for the following non-autonomous evolutionary equations with a fading memory

$$
u_{t t}-\Delta u_{t}-\Delta u-\Delta u_{t t}(t)-\int_{0}^{\infty} \mu(s) \Delta \eta^{t}(s) d s+f(u)=g
$$

and

$$
\eta_{t}^{t}=-\eta_{s}^{t}+u_{t}
$$

The problem is supplemented with the boundary condition

$$
\left.u(x, t)\right|_{\partial \Omega}=0 \quad \text { for all } \quad t \geq \tau, \tau \in R
$$

and initial condition

$$
u(x, t)=u_{\tau}(x, t), u_{t}(x, t)=\frac{\partial}{\partial t} u_{\tau}(x, t) \quad \mathrm{t} \leq \tau, \tau \in R
$$

Where $\Omega$ is a bounded smooth domain in $R^{3}, g=g(t)$ is a given external time-dependent forcing, $f$ is the critical nonlinearity.

Problem(1)is related to the following equations like

$$
u_{t t}-u_{x x t}-u_{x x}-u_{x x t t}=0 \text {, }
$$

Which appear as a class of nonlinear evolution equations, and that is used to represent the propagation problems of lengthways-wave in nonlinear elastic rods and Ion-sonic of space transformation by weak nonlinear effect (see for instance[1,3]). Since (1) contain terms $\Delta u_{t t}$, it is essentially different from D’Alembert wave equation.

Let us recall some results concerning the problem (1). In $[10,11]$ etc, authors studied this equations with Dirichlet boundary conditions as $\mu=0$. Recently, Araújo et al.[5] and M. Conti [4], H. Yassine and A. Abbas [9] studied the well posedness for this equations. In particular, Qin[8] obtain the existence of uniform attractors as $f=0$.

Maybe, we could establish the existence of uniform attractors of (1) using the method in [16, 17], but the regularity and structure cannot obtain directly. In this paper, we will apply the techniques introduced in Sun [14] to overcome the difficulty due to the critical nonlinearity, and establish the asymptotic regularity of the solutions. Based on this regularity result, we obtain the asymptotic compactness of the non-autonomous system and prove the existence of a uniform attractor together with its structure in $H_{0}^{1}(\Omega) \times H_{0}^{1}(\Omega) \times L_{\mu}^{2}\left(R^{+} ; H_{0}^{1}(\Omega)\right)$. It is noteworthy that the compact uniform attractor is bounded in $D(A) \times D(A) \times L_{\mu}^{2}\left(R^{+} ; D(A)\right)$.

For conveniences, hereafter let $|u|$ be the modular (or absolute value) of $u$ and $|\cdot|_{p}$ be the norm of $L^{P}(\Omega)(P>1)$. Denote $H^{-1}(\Omega)$ is the dual space of $H_{0}^{1}(\Omega)$ and $\|\cdot\|_{H^{-1}}$ be the norm of $H^{-1}(\Omega)$.Let $\left(v,\|\cdot\|_{v}\right)$ be a Banach space, we denote respectively the inner product and norm of the weighted space $L_{\mu}^{2}\left(R^{+} ; v\right)$ by

$$
\langle\varphi, \psi\rangle_{\mu, v}=\int_{0}^{\infty} \mu(s)\langle\varphi(s), \psi(s)\rangle_{\nu} d s
$$

and

$$
\|\varphi\|_{\mu, v}^{2}=\int_{0}^{\infty} \mu(s)\|\varphi(s)\|_{\nu}^{2} d s
$$


Denote $A=-\Delta$ with domain $D(A)=H^{2}(\Omega) \cap H_{0}^{1}(\Omega)$, and for $r \in R$, let $\varepsilon_{r}=D\left(A^{\frac{r+1}{2}}\right)$ and $\|\cdot\|_{r}$ be the norm of $\varepsilon_{r}$. We also define the system state space for $\left(u, u_{t}, \eta\right)$ as $H_{r}$, together with a dense subspace $M$ :

$$
\begin{gathered}
H_{r}=\varepsilon_{r} \times \mathcal{E}_{r} \times L_{\mu}^{2}\left(R^{+} ; \mathcal{E}_{r}\right), \\
M=D(A) \times D(A) \times\left(L_{\mu}^{2}\left(R^{+} ; D(A)\right) \cap H_{\mu}^{2}\left(R^{+} ; H_{0}^{1}(\Omega)\right)\right) .
\end{gathered}
$$

We also define the norm of the product space $H_{r}$ as follows

$$
\|z\|_{H_{r}}^{2}=\left\|\left(u, v, \eta^{t}\right)\right\|_{H_{r}}^{2}=\frac{1}{2}\left(\|u\|_{r}^{2}+\|v\|_{r}^{2}+\left\|\eta^{t}\right\|_{u, \varepsilon_{r}}^{2}\right)
$$

for any $z=\left(u, v, \eta^{t}\right) \in H_{r}$.

Let $C$ be an arbitrarily positive constant, which may be differential from line to line, even in the same line.

For the memory kernel $\mu(s)$, we assume the following hypotheses: for all $s \in R^{+}$and some $\delta>0$

$$
\begin{gathered}
\mu \in C^{1}\left(R^{+}\right) \cap L^{1}\left(R^{+}\right), \quad \mu(\mathrm{s}) \geq 0, \quad \mu^{\prime}(\mathrm{s}) \leq 0, \\
\mu^{\prime}(\mathrm{s})+\delta \mu(\mathrm{s}) \leq 0
\end{gathered}
$$

We introduce a new variable of the system,

$$
\eta=\eta^{t}(x, s):=u(x, t)-u(x, t-s), \quad s \in R^{+},
$$

which will be ruled by a supplementary equation. Denoting

$$
\eta_{t}^{t}=\frac{\partial}{\partial t} \eta^{t}, \quad \eta_{s}^{t}=\frac{\partial}{\partial s} \eta^{t}
$$

Then the following estimate holds(See[17])

$$
\left\langle\eta^{t}, \eta_{s}^{t}\right\rangle_{\mu, v} \geq \frac{\delta}{2}\left\|\eta^{t}\right\|_{\mu, v}^{2}
$$

The past history $u_{\tau}(\tau-s)$ of the variable $u$ satisfies the condition as follows: there exist two positive constants $\Re$ and $\kappa \leq \delta$ such that

$$
\int_{0}^{\infty} e^{-\kappa s}\left\|u_{\tau}(\tau-s)\right\|_{0}^{2} d s \leq \mathfrak{R}
$$

The nonlinearity $f \in C^{1}(R, R)$, fulfills $f(0)=0$ satisfies the following decomposition

$$
\left|f^{\prime}(s)\right| \leq c\left(1+|s|^{4}\right) \text { for all } s \in R
$$

and

$$
\liminf _{|s| \rightarrow \infty} \frac{f(s)}{s}>-\lambda_{1}
$$

for any $s \in R$, where $c, \lambda_{1}$ are positive constants and $\lambda_{1}$ is the first eigenvalue of $-\Delta$ in $H_{0}^{1}(\Omega)$ with the Dirichlet boundary condition.

Calling $F(s)=\int_{0}^{s} f(y) d y$. Notice that by (8), the following inequalities hold for some $0<\lambda<\lambda_{1}$ and $c_{0} \geq 0$

$$
2 \int_{\Omega} f(u) u \geq 2 \int_{\Omega} F(u)-\lambda|u|_{2}^{2}-c_{0}
$$

For the time-dependent forcing $g$, we assume the following hypotheses: $g \in L_{b}^{2}\left(R ; L^{2}(\Omega)\right.$ ) (translation bounded in $\left.L_{w, l o c}^{2}\left(R ; L^{2}(\Omega)\right)\right)$, and with the norm

$$
\|g\|_{L_{b}^{2}}^{2}=\sup _{t \in R} \int_{t}^{t+1}|g(s)|_{2}^{2} d s<\infty .
$$

\section{PReliminaries}

We will complete our task exploiting the transitivity property of exponential attraction[15], that we recall below for the readers convenience.

Lemma 2.1.[15] Let $(H ; d)$ be an abstract metric space, $U(t ; \tau)$ be a Lipschitz continuous dynamical process in $H$, i.e

$$
\left\|U(t+\tau, \tau) z_{1}-U(t+\tau, \tau) z_{2}\right\|_{H} \leq L_{0} e^{v_{0} t}\left\|z_{1}-z_{2}\right\|_{H},
$$

for appropriate constants $v_{0} \geq 0$ and $L_{0} \geq 0$ which are independent of $z_{i}, \tau$ and $t$. We further assume that there exist three subsets $\boldsymbol{\kappa}_{1}, \boldsymbol{\kappa}_{2}, \boldsymbol{\kappa}_{3} \subset H$ such that

$$
\begin{aligned}
& \operatorname{dist}_{H}\left(U(t+\tau, \tau) \boldsymbol{K}_{1}, \boldsymbol{K}_{2}\right) \leq L_{1} e^{-v_{1} t}, \\
& \operatorname{dist}_{H}\left(U(t+\tau, \tau) \boldsymbol{K}_{2}, \boldsymbol{K}_{3}\right) \leq L_{2} e^{-v_{2} t},
\end{aligned}
$$

for some $v_{1}, v_{2} \geq 0$ and $L_{1}, L_{2} \geq 0$. Then it follows that

$$
\operatorname{dist}_{H}\left(U(t+\tau, \tau) \kappa_{1}, \kappa_{3}\right) \leq L e^{-v t},
$$

where $v=\frac{v_{1} v_{2}}{v_{0}+v_{1}+v_{2}}$ and $L=L_{0} L_{1}+L_{2}$

Lemma 2.2. [12] Let $X \subset \subset H \subset Y$ be Banach spaces, with $X$ reflexive. Suppose that $u_{n}$ is a sequence that is uniformly bounded in $L^{2}(0, T ; X)$ and $\frac{d u_{n}}{d t}$ is uniformly bounded in $L^{p}(0, T ; Y)$, for some $p>1$. Then there is a subsequence of $u_{n}$ that converges strongly in $L^{2}(0, T ; H)$. 


\section{UNIFORM ATTRACTOR IN $H_{0}$}

Throughout the paper, we assume $g_{0} \in L_{b}^{2}\left(R ; L^{2}(\Omega)\right)$ and $\sum$ is the hull of $g_{0}$ in $L_{w, l o c}^{2}\left(R ; L^{2}(\Omega)\right)$ and $g \in \sum$. Assume further that (2)-(3) and (6)-(8).

\section{A. The Well-Posedness}

By the standard Faedo-Galerkin methods, it easy to obtain the following result.

Lemma 3.1. for any $T>0$ and $z_{\tau}=\left(u_{\tau}, v_{\tau}, \eta^{\tau}\right) \in H_{0}$. problem (1.1) admits a unique week solution

$$
z=\left(u(x, t), u_{t}(x, t), \eta^{t}\right) \in C\left([\tau, T], H_{0}\right),
$$

satisfying

$$
\begin{aligned}
& u \in L^{\infty}\left(R_{\tau} ; H_{0}^{1}(\Omega)\right), u_{t} \in L^{\infty}\left(R_{\tau} ; H_{0}^{1}(\Omega)\right) \\
& u_{t t} \in L^{2}\left([\tau, T] ; H_{0}^{1}(\Omega)\right), \eta \in L^{\infty}\left(R_{\tau} ; L_{\mu}^{2}\left(R^{+} ; H_{0}^{1}(\Omega)\right)\right)
\end{aligned}
$$

The proof of Lemma3.1 is similar to that of Theorem 2.1 of Araújo et al.[5] and hence is omitted.

Form Lemma 3.1 above, for each $g \in L_{b}^{2}\left(R ; L^{2}(\Omega)\right)$ we define a process

$$
\begin{aligned}
& U_{g}(t, \tau): H_{0} \rightarrow H_{0}, \\
& z_{\tau}=\left(u_{\tau}, v_{\tau}, \eta^{\tau}\right) \rightarrow\left(u(t), v(t), \eta^{t}\right)=U_{g}(t, \tau) z_{\tau} .
\end{aligned}
$$

\section{B. Dissipativity}

First of all, we can obtain the following theorem from [4]

Theorem 3.2.There exists a positive constant $M_{0}$ with following property: given any $Y \geq 0$ there exist $T_{0}=T_{0}(Y, \tau) \geq \tau$ such that, whenever $\left\|z_{\tau}\right\|_{H_{0}} \leq Y$ it follows that

$$
\left\|U_{g}(t, \tau) z_{\tau}\right\|_{H_{0}}^{2} \leq M_{0}, \quad \forall t \geq T_{0} .
$$

Consequently, the set

$$
B_{0}=\left\{z_{\tau} \in H_{0}:\left\|z_{\tau}\right\|_{H_{0}}^{2} \leq M_{0}\right\}
$$

is a bounded uniformly (w.r.t $\sigma \in \sum$ ) absorbing set for $U_{g}(t, \tau)$ on $H_{0}$, that is, for any bounded (in $H_{0}$ ) subsets $B$,there is a $T_{0}=T_{0}\left(\|B\|_{H_{0}}, \tau\right) \geq \tau$ such that

$$
U_{g \in \Sigma} U_{g}(t, \tau) B \subset B_{0}
$$

for every $t \geq T_{0}$.
Combining Lemma 3.1, we know that for any $\tau \in R, U_{g}$ maps the bounded set of $H_{0}$ into a bounded set of $H_{0}$ for all $t \geq \tau$, that is

Corollary 3.3.Given any $R>0$, there is $M_{R}=M_{R}\left(R,\|g\|_{L_{b}^{2}}\right)$ such that for all $\left\|z_{\tau}\right\|_{H_{0}} \leq R$,

$$
\left\|U_{g}(t, \tau) z_{\tau}\right\|_{H_{0}}^{2} \leq M_{R}, \forall t>\tau
$$

Lemma 3.4. Given any $R>0$, let $z_{1 \tau}, z_{2 \tau} \in H_{0}$ $g_{1}, g_{2} \in L_{b}^{2}\left(R ; L^{2}(\Omega)\right)$,be two initial data, and $\left\|z_{i \tau}\right\|_{H_{0}} \leq R(i=1,2)$.Then the following estimate holds,

$$
\left\|U_{g_{1}}(t, \tau) z_{1 \tau}-U_{g_{2}}(t, \tau) z_{2 \tau}\right\|_{H_{0}}^{2} \leq Q(R) e^{k(t-\tau)}\left(\left\|z_{1 \tau}-z_{2 \tau}\right\|_{H_{0}}^{2}+\left\|g_{1}-g_{2}\right\|_{L_{b}^{2}}^{2}\right)
$$

for any $t \geq \tau$ and some $k=k(R)$.

\section{Asymptotic Regularity}

For the nonlinear function $f(u)$ from[2], we know that $f$ has the following decomposition

$$
f=f_{0}+f_{1}
$$

where $f_{0}, f_{1} \in C(R)$ and satisfy

$$
\begin{gathered}
f_{0}(s) s \geq 0 \text { for all } s \in R \\
\left|f_{0}(s)\right| \leq c\left(1+|s|^{5}\right) \text { for all } s \in R \\
\left|f_{1}(s)\right| \leq c\left(1+|s|^{\gamma}\right) \text { for all } s \in R \text { with some } \gamma<5, \\
\liminf _{|s| \rightarrow \infty} \frac{f_{1}(s)}{s}>-\lambda_{1}
\end{gathered}
$$

where $c, \lambda_{1}$ are positive constants and $\lambda_{1}$ is the first eigenvalue of $-\Delta$ in $H_{0}^{1}(\Omega)$ with the Dirichlet boundary condition. Denote

$$
\sigma=\min \left\{\frac{1}{4}, \frac{5-\gamma}{2}\right\}
$$

In order to obtain the regularity estimates later, we decompose the solution $U_{g}(t, \tau) z_{\tau}=\left(u(t), u_{t}(t), \eta^{t}\right)$ into the sum:

$$
U_{g}(t, \tau) z_{\tau}=S(t, \tau) z_{\tau}+K_{g}(t, \tau) z_{\tau}
$$

$S(t, \tau) z_{\tau}=\left(v(t), v_{t}(t), \xi^{t}\right), K_{g}(t, \tau) z_{\tau}=\left(w(t), w_{t}(t), \zeta^{t}\right)$ are the solutions the following equations respectively 


$$
\left\{\begin{array}{l}
v_{t t}-\Delta v_{t t}-\Delta v_{t}-\Delta v-\int_{0}^{\infty} \mu(s) \Delta \xi^{t}(s) d s+f_{0}(v)=0 \\
\xi_{t}^{t}=-\xi_{s}^{t}+v_{t}(t), \\
\left(v(\tau), v_{t}(\tau), \xi^{\tau}\right)=z_{\tau},\left.\quad v\right|_{\partial \Omega}=0,\left.\xi\right|_{\partial \Omega \times R^{+}}=0
\end{array}\right.
$$

and

$$
\left\{\begin{array}{l}
w_{t t}-\Delta w_{t t}-\Delta w_{t}-\Delta w-\int_{0}^{\infty} \mu(s) \Delta \zeta^{t}(s) d s+f(u)-f_{0}(v)=g(x, t), \\
\zeta_{t}^{t}=-\zeta_{s}^{t}+w_{t}(t), \\
\left(w(\tau), w_{t}(\tau), \zeta^{\tau}\right)=0,\left.w\right|_{\curvearrowright 2}=0,\left.\zeta\right|_{22 \times R^{t}}=0 .
\end{array}\right.
$$

We will establish a priori estimates about the solutions of (16) and(17), which are the basis of our works.

Lemma 3.5.For any initial data $z_{\tau} \in H_{0}$, the solutions of (16) satisfy the following estimates: There exists constant $k_{0}$ such that for every $t \geq \tau$,

$$
\left\|S(t, \tau) z_{\tau}\right\|_{H_{0}}^{2}=\|v(t)\|_{0}^{2}+\left\|v_{t}(t)\right\|_{0}^{2}+\left\|\xi^{t}\right\|_{\mu, \varepsilon_{0}}^{2} \leq Q_{1}\left(\left\|z_{\tau}\right\|_{H_{0}}\right) e^{-k_{0}(t-\tau)}
$$
where $Q_{1}(\cdot)$ is an increasing function on $[0, \infty), Q_{1}$ and $k_{0}$ only depend on the $H_{0}$ - bound of $z_{\tau}$, but both are independent of $\tau$.

Proof Repeating word by word the proof of Theorem 3.2, that applies to the present case with $S(t, \tau) z_{\tau}$ in place of $U_{g}(t, \tau) z_{\tau}$ (with the further simplification that $C=0$, for now $f_{1} \equiv 0$ and $g \equiv 0$ ), It follows that

$$
\left\|S(t, \tau) z_{\tau}\right\|_{H_{0}}^{2}=\|v(t)\|_{0}^{2}+\left\|v_{t}(t)\right\|_{0}^{2}+\left\|\xi^{t}\right\|_{\mu, \varepsilon_{0}}^{2} \leq Q_{1}\left(\left\|z_{\tau}\right\|_{H_{0}}\right) e^{-k_{0}(t-\tau)}
$$

For the solution of (17), we have

Lemma 3.6.For any $\tau \in R$, the solutions of (17) satisfy the following estimates: There exists constant $k_{1}$ such that for every $t \geq \tau$,

$$
\begin{aligned}
\left\|K_{g}(t, \tau) z_{\tau}\right\|_{H_{\sigma}}^{2} & =\|w(t)\|_{\sigma}^{2}+\left\|w_{t}(t)\right\|_{\sigma}^{2}+\left\|\xi^{t}\right\|_{\mu_{,} \varepsilon_{\sigma}}^{2}, \\
& \leq Q_{2}\left(\left\|z_{\tau}\right\|_{H_{0}}\right) e^{k_{1}(t-\tau)}\left(1+\|g\|_{L_{b}^{2}}^{2}\right)
\end{aligned}
$$

where $Q_{2}(\cdot)$ is an increasing function on $[0, \infty)$, and $\sigma$ is given in (15).

Proof. Multiplying(17) by $A^{\sigma} w_{t}(t)$, and integrating in $d x$ over $\Omega$,we get that

$$
\begin{aligned}
& \frac{1}{2} \frac{d}{d t}\left(\left|A^{\frac{\sigma}{2}} w_{t}(t)\right|_{2}^{2}+\|w(t)\|_{\sigma}^{2}+\left\|w_{t}(t)\right\|_{\sigma}^{2}\right)+\left\|w_{t}(t)\right\|_{\sigma}^{2}-\left\langle\int_{0}^{\infty} \mu(s) \Delta \zeta^{t}(s) d s, A^{\sigma} w_{t}(t)\right\rangle \\
& =-\left\langle f(u)-f_{0}(v), A^{\sigma} w_{t}(t)\right\rangle+\left\langle A^{\sigma} w_{t}(t), g(x, t)\right\rangle .
\end{aligned}
$$

Similar to that in Theorem 3.2 above, we get

$$
-\int_{\Omega} \int_{0}^{\infty} \mu(s) \Delta \zeta^{t}(s) A^{\sigma} \zeta_{t}(s) d s d x=\frac{1}{2} \frac{d}{d t}\left\|\zeta^{t}\right\|_{\mu, \varepsilon_{\sigma}}^{2},
$$

and

$$
-\int_{\Omega} \int_{0}^{\infty} \mu(s) \Delta \zeta^{t}(s) A^{\sigma} \zeta_{s}^{t}(s) d s d x \geq \frac{\delta}{2}\left\|\zeta^{t}\right\|_{\mu, \varepsilon_{\sigma}}^{2}
$$

Next we deal with the nonlinearity, we have

$$
\left|\left\langle f(u)-f_{0}(v), A^{\sigma} w_{t}(t)\right\rangle\right| \leq\left|\left\langle f(u)-f(v), A^{\sigma} w_{t}(t)\right\rangle\right|+\left|\left\langle f_{1}(v), A^{\sigma} w_{t}(t)\right\rangle\right|
$$
and by Corollary (3.3) and Lemma (3.5), we have

$$
\|u(t)\|_{0}^{2}+\|v(t)\|_{0}^{2} \leq M_{1} \text { for all } t \geq \tau,
$$

where the constant $M_{1}$ depends on $\left\|Z_{\tau}\right\|_{H_{0}}$ but independent of $\tau$.

From (7), (21) and Hölder's inequality, then we have

$$
\left|\left\langle f(u)-f(v), A^{\sigma} w_{t}(t)\right\rangle\right| \leq C_{M_{1}}\|w(t)\|_{\sigma}^{2}+\frac{1}{4}\left\|w_{t}(t)\right\|_{\sigma}^{2} .
$$

Note that $\sigma \leq \frac{5-\gamma}{2}$, so we can get the following estimates

$$
\left|\left\langle f_{1}(v), A^{\sigma} w_{t}(t)\right\rangle\right| \leq C+\frac{1}{4}\left\|w_{t}(t)\right\|_{\sigma}^{2} .
$$

Moreover,

$$
\left|\left\langle A^{\sigma} w_{t}(t), g(t)\right\rangle\right| \leq C|g(t)|_{2}^{2}+\frac{1}{2}\left\|w_{t}(t)\right\|_{\sigma}^{2} .
$$

Combined with (19)-(20) and(22)-(23), by (18), we have that

$$
\frac{d}{d t}\left(\left|A^{\frac{\sigma}{2}} w_{t}(t)\right|_{2}^{2}+\left\|w_{t}(t)\right\|_{\sigma}^{2}+\|w(t)\|_{\sigma}^{2}+\left\|\zeta^{t}\right\|_{\mu, \varepsilon_{\sigma}}^{2}\right) \leq C\left(1+|g(t)|_{2}^{2}\right)+C_{M_{1}}\|w(t)\|_{\sigma}^{2} .
$$

Applying the Gronwall's inequality, we deduce that

$$
\begin{aligned}
& \left|A^{\frac{\sigma}{2}} w_{t}(t)\right|_{2}^{2}+\left\|w_{t}(t)\right\|_{\sigma}^{2}+\|w(t)\|_{\sigma}^{2}+\left\|\zeta^{t}\right\|_{\mu, \varepsilon_{\sigma}}^{2} \\
\leq & \mathrm{Q}_{2}\left(\left\|z_{\tau}\right\|_{H_{0}}\right) e^{k_{1}(t-\tau)}\left(1+\|g\|_{L_{b}^{2}}^{2}\right)
\end{aligned}
$$


here $k_{1}=C_{M_{1}}$ and $C_{M_{1}}$ depend on $\left\|z_{\tau}\right\|_{H_{0}}$.

Lemma 3.7.For any $\varepsilon>0 u_{t}(t)$ is decomposed as

$$
u(t)=v_{1}(t)+w_{1}(t),
$$

$v_{1}(t)$ satisfies: there is a positive constant $M_{1}=M_{1}\left(\left\|z_{\tau}\right\|_{\varepsilon_{0}}\right)$ such that the following estimates are true

$$
\left\|v_{1}(t)\right\|_{0}^{2} \leq M_{1}
$$

and

$$
\int_{s}^{t}\left\|v_{1}(v)\right\|_{0}^{2} d v \leq \varepsilon(t-s)+C_{\varepsilon} \text { for all } t \geq s \geq \tau
$$

As well as $w_{1}(t)$ satisfies the following estimate

$$
\left\|w_{1}(t)\right\|_{\sigma}^{2} \leq K_{\varepsilon} \text { for all } t \geq \tau
$$

with the constants $C_{\varepsilon}$ and $K_{\varepsilon}$ depending on $\varepsilon,\left\|z_{\tau}\right\|_{H_{0}}$ and $\|g\|_{L_{b}^{2}}$, but both being independent of $\tau$.

The proof of this lemma is similar to that in Sun [14].

In what follows we begin to establish the asymptotic regularity of the solutions of (1).

Lemma 3.8. There exists constant $\Upsilon_{0}$ which depends only on the $H_{0}$-bounds of $B\left(\subset H_{0}\right)$, such that for any $\tau \in R$

$$
\left\|K_{g}(t, \tau) z_{\tau}\right\|_{H_{\sigma}}^{2} \leq \Upsilon_{0} \text { for all } t \geq \tau \text { and } z_{\tau} \in B \text {, }
$$

where $\sigma$ is given in (15).

Proof. Taking inner product of the first equation of (17) and $A^{\sigma}\left(w_{t}+\varepsilon w\right)$ ( $\varepsilon$ is an positive undetermined constant), we get that

$$
\begin{aligned}
& \left\langle w_{t t}-\Delta w-\Delta w_{t}-\Delta w_{t t}-\int_{0}^{t} \mu(s) \Delta \zeta^{t}(s) d s, A^{\sigma}\left(w_{t}+\varepsilon w\right)\right\rangle \\
= & -\left\langle f(u)-f_{0}(v), A^{\sigma}\left(w_{t}+\varepsilon w\right)\right\rangle+\left\langle g(x, t), A^{\sigma}\left(w_{t}+\varepsilon w\right)\right\rangle,
\end{aligned}
$$

In the following, we will deal with the left side of (27) one by one. Similar to that (19) and (20), we get that

$$
-\left\langle\int_{0}^{\infty} \mu(s) \Delta \zeta^{t}(s) d s, A^{\sigma}\left(w_{t}+\varepsilon w\right)\right\rangle \geq \frac{1}{2} \frac{d}{d t}\left\|\zeta^{t}\right\|_{\mu, \varepsilon_{\sigma}}^{2}+\frac{\delta}{2}\left\|\zeta^{t}\right\|_{\mu, \varepsilon_{\sigma}}^{2}-\varepsilon\left\|\zeta^{t}\right\|_{\mu, \varepsilon_{\sigma}}\|W\|_{\sigma} .
$$

Now we rewrite (27) as

$$
\frac{d}{d t} E_{2}(t)+I_{2}(t)=-\left\langle f(u)-f_{0}(v), A^{\sigma}\left(w_{t}+\varepsilon w\right)\right\rangle+\left\langle g(x, t), A^{\sigma}\left(w_{t}+\varepsilon w\right)\right\rangle
$$

here

$$
\begin{gathered}
E_{2}(t)=\frac{1}{2}\left|A^{\frac{\sigma}{2}} w_{t}\right|_{2}^{2}+\varepsilon\left\langle w_{t}, A^{\sigma} w\right\rangle+\frac{1+\varepsilon}{2}\|w\|_{\sigma}^{2}+\frac{1}{2}\left\|\zeta^{t}\right\|_{\mu, \varepsilon_{\sigma}}^{2} \\
+\frac{1}{2}\left\|w_{t}\right\|_{\sigma}^{2}+\varepsilon\left\langle A w_{t}, A^{\sigma} w\right\rangle
\end{gathered}
$$

and

$$
\begin{aligned}
I_{2}(t) & =-\varepsilon\left|A^{\frac{\sigma}{2}} w_{t}\right|_{2}^{2}+\varepsilon\|w\|_{\sigma}^{2}+\left\|w_{t}\right\|_{\sigma}^{2}-\varepsilon\left\|w_{t}\right\|_{\sigma}^{2} \\
& +\frac{\delta}{2}\left\|\zeta^{t}\right\|_{\mu, \varepsilon_{\sigma}}^{2}-\varepsilon\left\|\zeta^{t}\right\|_{\mu, \varepsilon_{\sigma}}^{2}\|w\|_{\sigma}
\end{aligned}
$$

Applying the Hölder's inequality in (29), we get that

$$
E_{2}(t) \leq \alpha_{1}\left(\left\|w_{t}\right\|_{\sigma}^{2}+\|w\|_{\sigma}^{2}+\left\|\zeta^{t}\right\|_{\mu, \varepsilon_{\sigma}}^{2}\right)
$$

where $\alpha_{1}=\max \left\{\frac{1+\varepsilon}{2}\left(1+\frac{1}{\lambda_{1}}\right), \frac{\varepsilon}{2 \lambda_{1}}+\frac{1+2 \varepsilon}{2}\right\}$.

On the other hand, we have

$$
E_{2}(t) \geq \frac{1}{2}\left(1-\frac{\varepsilon^{2}}{\lambda_{1}}\right)\|w\|_{\sigma}^{2}+\frac{1}{2}(1-\varepsilon)\left\|w_{t}\right\|_{\sigma}^{2}+\frac{1}{2}\|\zeta\|_{\mu, \varepsilon_{\sigma}}^{2}
$$

choose

$$
\varepsilon \leq \frac{1}{2} \min \left\{1, \sqrt{\lambda_{1}}\right\}
$$

$$
\begin{aligned}
& \text { Let } \beta_{1}=\min \left\{\frac{1}{2}(1-\varepsilon), \frac{1}{2}\left(1-\frac{\varepsilon^{2}}{\lambda_{1}}\right)\right\}>0 \text {, then } \\
& \qquad E_{2}(t) \geq \beta_{1}\left(\left\|w_{t}\right\|_{\sigma}^{2}+\|w\|_{\sigma}^{2}+\left\|\zeta^{t}\right\|_{\mu, \varepsilon_{\sigma}}^{2}\right) .
\end{aligned}
$$

Toward $I_{2}(t)$, we have

$$
I_{2}(t) \geq \frac{\varepsilon}{2}\|w\|_{\sigma}^{2}+\left(1-\left(1+\frac{1}{\lambda_{1}}\right) \varepsilon\right)\left\|w_{t}\right\|_{\sigma}^{2}+\frac{1}{2}(\delta-\varepsilon)\left\|\zeta^{t}\right\|_{\mu, \varepsilon_{\sigma}}^{2}
$$

Combined with (31), choose

$$
\varepsilon=\frac{1}{2} \min \left\{\frac{\lambda_{1}}{1+\lambda_{1}}, \delta, \sqrt{\lambda_{1}}\right\}
$$

Let $\alpha_{2}=\frac{1}{2} \min \left\{\varepsilon, 2\left(1-\left(1+\frac{1}{\lambda_{1}}\right) \varepsilon\right), \delta-\varepsilon\right\}$.

$$
I_{2} \geq \alpha_{2}\left(\left\|w_{t}\right\|_{\sigma}^{2}+\|w\|_{\sigma}^{2}+\left\|\zeta^{t}\right\|_{\mu, \varepsilon_{\sigma}}^{2}\right)
$$


From Corollary 3.3 and Lemma 3.5, there is a positive constant $M_{2}=M_{2}\left(\left\|z_{\tau}\right\|_{\varepsilon_{0}}\right)$ such that

$$
\left\|\kappa_{g}(t, \tau) z_{\tau}\right\|_{\varepsilon_{0}}^{2} \leq M_{2}
$$

holds for any $\tau \in R$.

Since $\frac{1+\delta}{2}<1$, employing the interpolation inequality, we can get that

$$
\left|\left\langle g(t), A^{\sigma}\left(w_{t}+\varepsilon w\right)\right\rangle\right| \leq C_{\alpha_{2}}|g(t)|_{2}^{2}+\frac{\alpha_{2}}{8}\left(\left\|w_{t}\right\|_{\sigma}^{2}+\|w\|_{\sigma}^{2}\right)
$$

and employing Lemma 3.7 to deal with the nonlinear term:

$$
\begin{aligned}
& \left|\left\langle f(u)-f_{0}(v), A^{\sigma}\left(w_{t}+\varepsilon w\right)\right\rangle\right| \\
& \leq\left|\left\langle f(u)-f(v), A^{\sigma}\left(w_{t}+\varepsilon w\right)\right\rangle\right|+\left|\left\langle f_{1}(v), A^{\sigma}\left(w_{t}+\varepsilon w\right)\right\rangle\right| .
\end{aligned}
$$

From (7) and Lemma 3.5, we have

$$
\begin{aligned}
& \left|\left\langle f(u)-f(v), A^{\sigma}\left(w_{t}+\varepsilon w\right)\right\rangle\right| \\
& \leq C M_{2}+C \int_{\Omega}\left(\left.u(t)\right|^{4}+|v(t)|^{4}\right)|w(t)|\left|A^{\sigma}\left(w_{t}+\varepsilon w\right)\right|
\end{aligned}
$$

Using Lemma 3.7, we have

$$
\int_{\Omega}|u(t)|^{4}|w(t)|\left|A^{\sigma}\left(w_{t}+\varepsilon w\right)\right| \leq \int_{\Omega}\left(\left|v_{1}(t)\right|^{4}+\left|w_{1}(t)\right|^{4}\right)|w(t)|\left|A^{\sigma}\left(w_{t}+\varepsilon w\right)\right|
$$

and

$$
\int_{\Omega}\left|v_{1}\right|^{4}|w|\left|A^{\sigma}\left(w_{t}+\varepsilon w\right)\right| \leq \mathrm{M}_{1}\left\|v_{1}\right\|_{0}^{2}\left(\left\|w_{t}\right\|_{\sigma}^{2}+\|w\|_{\sigma}^{2}\right) .
$$

Therefore, note that $\sigma=\min \left\{\frac{1}{4}, \frac{5-\gamma}{2}\right\}$,we have $\frac{12}{5}<\frac{6}{1+6 \sigma}<6$, and then

$$
\int_{\Omega}\left|w_{1}\right|^{4}|w|\left|A^{\sigma}\left(w_{t}+\varepsilon w\right)\right| \leq \frac{2 K_{\varepsilon}^{8} M_{2}^{2}}{\alpha_{2}}+\frac{\alpha_{2}}{4}\left(\left\|w_{t}\right\|_{\sigma}^{2}+\|w\|_{\sigma}^{2}\right)
$$

where $K_{\varepsilon}$ is given in (26).

$$
\int_{\Omega}|\nu|^{4}|w|\left|A^{\sigma}\left(w_{t}+\varepsilon w\right)\right| \leq 2 Q_{1}\left(\left\|Z_{\tau}\right\|_{H_{0}}\right)\|v\|_{0}^{2}\left(\left\|w_{t}\right\|_{\sigma}^{2}+\|w\|_{\sigma}^{2}\right) .
$$

where $\mathrm{Q}_{1}\left(\left\|Z_{\tau}\right\|_{H_{0}}\right)$ from Lemma 3.5.

Substitute (40)-(43) into (39), we get that

$$
\begin{aligned}
\left\langle\left\langle f(u)-f(v), A^{\sigma}\left(w_{t}+\varepsilon w\right)\right\rangle\right| & \leq C\left(\|v\|_{0}^{2}+\left\|v_{1}\right\|_{0}^{2}\right)\left(\left\|w_{t}\right\|_{\sigma}^{2}+\|w\|_{\sigma}^{2}\right) \\
& +\frac{\alpha_{2}}{4}\left(\left\|w_{t}\right\|_{\sigma}^{2}+\|w\|_{\sigma}^{2}\right)+K_{1}
\end{aligned}
$$

where $K_{1}=C M_{2}+\frac{2 C K_{\varepsilon}^{8} M_{2}^{2}}{\alpha_{2}}$.

Similarly,

$$
\left|\left\langle f_{1}(v), A^{\sigma}\left(w_{t}+\varepsilon w\right)\right\rangle\right| \leq K_{2}+\frac{\alpha_{2}}{8}\left(\left\|w_{t}\right\|_{\sigma}^{2}+\|w\|_{\sigma}^{2}\right)
$$

Moreover, it follows Lemma 3.7,

$$
\int_{\tau}^{\infty}\|v(s)\|_{0}^{2} d s \leq \frac{\mathrm{Q}_{1}\left(\left\|z_{\tau}\right\|_{H_{0}}\right)}{k_{0}}
$$

then for any $\varepsilon>0$

$$
\int_{s}^{t}\left(\|v(s)\|_{0}^{2}+\left\|v_{1}(s)\right\|_{0}^{2}\right) d s \leq \varepsilon(t-s)+\frac{\mathrm{Q}_{1}\left(\left\|z_{\tau}\right\|_{H_{0}}\right)}{k_{0}}+C_{\varepsilon} .
$$

Hence, combining the above estimates into (28), we see that for all $t \geq \tau$,

$\frac{d}{d t} E_{2}(t)+\frac{\alpha_{2}}{2 \alpha_{1}} E_{2}(t) \leq \frac{C}{\beta_{1}}\left(\|v(t)\|_{0}^{2}+\left\|v_{1}(t)\right\|_{0}^{2}\right) E_{2}(t)+K_{1}+K_{2}$

Then Gronwall's inequality yields, for any $t \geq T>\tau$

$$
E_{2}(t) \leq \beta E_{2}(T) e^{-\gamma(t-T)}+\rho,
$$

here $\beta>0$ is a constant which depended on initial data and $\gamma, \rho$ are positive constants which depended on initial data.

At the last, by Lemma 3.6, (31), (34) and noting that $T>\tau$ is fixed, then the proof is completed.

Lemma 3.9.Assume $B_{\sigma}$ is bounded in $H_{\sigma}$.Then there exists a constant $M_{\sigma}(>0)$ which only depends on the $H_{\sigma}$-bounds of $B_{\sigma}$ such that for any $\tau \in R$

$$
\left\|U_{g}(t, \tau) z_{\tau}\right\|_{H_{\sigma}} \leq M_{\sigma} \text { for all } t \geq \tau \text { and } z_{\tau} \in B_{\sigma}
$$

Proof Multiply (1) by $A^{\sigma}\left(u_{t}+\varepsilon u\right)(\varepsilon$ is a positive undetermined constant), we get that

$$
\frac{d}{d t} E_{3}(t)+I_{3}(t)=-\left\langle f(u), A^{\sigma}\left(u_{t}+\varepsilon u\right)\right\rangle+\left\langle g(x, t), A^{\sigma}\left(u_{t}+\varepsilon u\right)\right\rangle,
$$

here 


$$
\begin{aligned}
E_{3}(t) & =\frac{1}{2}\left|A^{\frac{\sigma}{2}} u_{t}\right|_{2}^{2}+\varepsilon\left\langle u_{t}, A^{\sigma} u\right\rangle+\frac{1+\varepsilon}{2}\|u\|_{\sigma}^{2} \\
& +\frac{1}{2}\left\|\xi^{t}\right\|_{\mu, \varepsilon_{\sigma}}^{2}+\frac{1}{2}\left\|u_{t}\right\|_{\sigma}^{2}+\varepsilon\left\langle A u_{t}, A^{\sigma} u\right\rangle,
\end{aligned}
$$

and

$$
\begin{gathered}
I_{3}(t)=-\varepsilon\left|A^{\frac{\sigma}{2}} u_{t}\right|_{2}^{2}+\varepsilon\|u\|_{\sigma}^{2}+\left\|u_{t}\right\|_{\sigma}^{2}-\varepsilon\left\|u_{t}\right\|_{\sigma}^{2} \\
+\frac{\delta}{2}\left\|\xi^{t}\right\|_{\mu, \varepsilon_{\sigma}}^{2}-\varepsilon\left\|\xi^{t}\right\|_{\mu, \varepsilon_{\sigma}}^{2}\|u\|_{\sigma} .
\end{gathered}
$$

Applying the Hölder's inequality in (48), we get that

$$
E_{3}(t) \leq \alpha_{1}\left(\left\|u_{t}\right\|_{\sigma}^{2}+\|u\|_{\sigma}^{2}+\left\|\xi^{t}\right\|_{\mu, \varepsilon_{\sigma}}^{2}\right)
$$

where $\alpha_{1}$ from (31).

On the other hand, we have

$$
E_{3}(t) \geq \frac{1}{2}\left(1-\frac{\varepsilon^{2}}{\lambda_{1}}\right)\|u\|_{\sigma}^{2}+\frac{1}{2}(1-\varepsilon)\left\|u_{t}\right\|_{\sigma}^{2}+\frac{1}{2}\left\|\xi^{t}\right\|_{\mu,,_{\sigma}}^{2}
$$

choose

$$
\varepsilon \leq \frac{1}{2} \min \left\{1, \sqrt{\lambda_{1}}\right\}
$$

then

$$
E_{3}(t) \geq \beta_{1}\left(\left\|u_{t}\right\|_{\sigma}^{2}+\|u\|_{\sigma}^{2}+\left\|\xi^{t}\right\|_{\mu, \varepsilon_{\sigma}}^{2}\right)
$$

where $\beta_{1}=\min \left\{\frac{1}{2}(1-\varepsilon), \frac{1}{2}\left(1-\frac{\varepsilon^{2}}{\lambda_{1}}\right)\right\}>0 \quad$ (from (34)).

Toward $I_{3}(t)$, we have

$$
I_{3}(t) \geq \frac{\varepsilon}{2}\|u\|_{\sigma}^{2}+\left(1-\left(1+\frac{1}{\lambda_{1}}\right) \varepsilon\right)\left\|u_{t}\right\|_{\sigma}^{2}+\frac{1}{2}(\delta-\varepsilon)\left\|\xi^{t}\right\|_{\mu, \varepsilon_{\sigma}}^{2}
$$

Combined with (52), choose

$$
\varepsilon=\frac{1}{2} \min \left\{\frac{\lambda_{1}}{1+\lambda_{1}}, \delta, \sqrt{\lambda_{1}}\right\} .
$$

Similar to (36), let $\alpha_{2}=\frac{1}{2} \min \left\{\varepsilon, 2\left(1-\left(1+\frac{1}{\lambda_{1}}\right) \varepsilon\right), \delta-\varepsilon\right\}$.

$$
I_{3} \geq \alpha_{2}\left(\left\|u_{t}\right\|_{\sigma}^{2}+\|u\|_{\sigma}^{2}+\left\|\xi^{t}\right\|_{\mu, \varepsilon_{\sigma}}^{2}\right) .
$$

From Corollary 3.3, there is a positive constant $Y=Y\left(\left\|z_{\tau}\right\|_{H_{0}}\right)$ such that

$$
\left\|U_{g}(t, \tau) z_{\tau}\right\|_{H_{0}}^{2} \leq Y
$$

holds for any $\tau \in R$.

Since $\frac{1+\delta}{2}<1$, employing the interpolation inequality, we can get that

$$
\left|\left\langle g(t), A^{\sigma}\left(u_{t}+\varepsilon u\right)\right\rangle\right| \leq C_{1}|g(t)|_{2}^{2}+\frac{\alpha_{2}}{6}\left(\left\|u_{t}\right\|_{\sigma}^{2}+\|u\|_{\sigma}^{2}\right) .
$$

where $C_{1}$ is a constant which depends on $\alpha_{2}$ and the measure of $\Omega$.Next, we deal with the nonlinear term,

$$
\left|\left\langle f(u), A^{\sigma} v\right\rangle\right| \leq c \int_{\Omega}\left(1+|u|^{5}\right)\left|A^{\sigma} v\right| \leq c \int_{\Omega}\left|A^{\sigma} v\right|+c \int_{\Omega}|u|^{5}\left|A^{\sigma} v\right| .
$$

Using the Hölder inequality and the Sobolev embedding theorem, it follows

$$
c \int_{\Omega}\left|A^{\sigma} v\right| \leq C_{2}+\frac{\alpha_{2}}{6}\left(\left\|u_{t}\right\|_{\sigma}^{2}+\|u\|_{\sigma}^{2}\right) .
$$

where $C_{1}$ is a constant which depends on $\alpha_{2}, c$ (from (7)) and the measure of $\Omega$

On the other hand, by Lemma 3.7, we get

$$
c \int_{\Omega}|u|^{5}\left|A^{\sigma} v\right| \leq C_{M}\left\|v_{1}\right\|_{0}^{2}\left(\left\|u_{t}\right\|_{\sigma}^{2}+\|u\|_{\sigma}^{2}\right)+\frac{\alpha_{2}}{6}\left(\left\|u_{t}\right\|_{\sigma}^{2}+\|u\|_{\sigma}^{2}\right)+C_{3} .
$$

where $C_{M}$ is a constant which depends on $c$ (from (7)) and the $H_{\sigma}$-bounds of initial data (see Corollary 3.3).

So we have

$$
\frac{d}{d t} E_{3}(t)+\frac{\alpha_{2}}{2 \alpha_{1}} E_{3}(t) \leq \frac{C_{M} \alpha_{2}}{\beta_{1}}\left\|v_{1}(t)\right\|_{0}^{2} E_{3}(t)+C_{1}|g(t)|_{2}^{2}+C_{2}+C_{3},
$$

where the positive constants $C_{i}, i=1,2,3$ depend on $\delta, K_{\varepsilon}$ and $\left\|z_{\tau}\right\|_{H_{0}}$.

Using Lemma 3.1 and integrating over $[\tau, T]$, we get that

$$
E_{3}(t) \leq \alpha_{1}\left\|z_{\tau}\right\|_{H_{\sigma}}^{2} e^{-w(t-\tau)+m_{1}}+\frac{m_{2} e^{w+m_{1}}}{1-e^{-w}}
$$


where

$$
w=\frac{\alpha_{2}}{4 \alpha_{1}}, m_{1}=C_{w}\left(\text { from (25)), } m_{2}=C_{2}+C_{3}+\|g\|_{L_{b}^{2}}^{2} .\right.
$$

We then complete the proof.

Lemma 3.10. For each $\theta \in[\sigma, 1]$, let $B$ be any bounded subset of $H_{\theta}$. Then there exists a constant $M_{\theta}$ which only depends on the $H_{\theta}$-bounds of $B$, such that for any $\tau \in R$,

$$
\left\|U_{g}(t, \tau) z_{\tau}\right\|_{H_{\theta}} \leq M_{\theta} \text { for all } t \geq \tau \text { and } z_{\tau} \in B
$$

Lemma 3.11. For each $\theta \in[\sigma, 1-\sigma]$, if the initial data set $B$ be any bounded subset of $H_{\theta}$, then the decomposed ingredient $\left(w(t), w_{t}(t)\right)$ (the solutions of (17)) satisfies, for any $\tau \in R$,

$$
\left\|K_{g}(t, \tau) z_{\tau}\right\|_{H_{\theta+\sigma}} \leq \Upsilon_{\theta} \quad \text { for all } t \geq \tau \text { and } z_{\tau} \in B \text {, }
$$

where the constant $\Upsilon_{\theta}$ only depends on the $H_{\theta}$-bounds of $B$.

Theorem 3.12.There exist a bounded (in $H_{1}$ ) set $B_{1} \subset H_{1}$, a positive constants $v$ and a monotonically increasing function $Q(\cdot)$ such that: For any bounded (in $H_{0}$ ) set $B \subset H_{0}$, any $g \in \sum, \tau \in R$ and $t \geq \tau$, the following estimate holds:

$$
\operatorname{dist}_{H_{0}}\left(U_{g}(t, \tau) B, B_{1}\right) \leq Q\left(\|B\|_{H_{0}}\right) e^{-v(t-\tau)} .
$$

where dist $_{H_{0}}(\cdot, \cdot)$ denotes the usual Hausdorff semi-distance in $H_{0}$.

Proof Let $B_{0}$ be the bounded uniformly (w.r.t $\sigma \in \sum$ ) absorbing set in $H_{0}$ (see Theorem 3.2).

$$
\begin{gathered}
\text { By Lemma } \quad 3.5 \quad \text { and Lemma } \quad 3.8 \text {, set } \\
A_{\sigma}=\left\{z \in H_{\sigma}:\|z\|_{H_{\sigma}} \leq \Upsilon_{0}\right\} \text { then } \\
\text { dist }_{H_{0}}\left(U_{g}(t, \tau) B_{0}, A_{\sigma}\right) \leq \text { dist }_{H_{0}}\left(S(t, \tau) B_{0}, A_{\sigma}\right) \leq Q_{1}\left(\left\|B_{0}\right\|_{H_{0}}\right) e^{-k_{0}(t-\tau)},
\end{gathered}
$$

where $\Upsilon_{0}$ is a constant from Lemma 3.8 corresponding to $B_{0}$.

Using $A_{\sigma}$ to replace $B_{0}$ in Lemma 3.11 and Lemma 3.5, then there is $A_{2 \sigma} \subset H_{0}$ which is bounded in $H_{2 \sigma}$ such that

$$
\operatorname{dist}_{H_{0}}\left(U_{g}(t, \tau) A_{\sigma}, A_{2 \sigma}\right) \leq \operatorname{dist}_{H_{0}}\left(S(t, \tau) A_{\sigma}, A_{2 \sigma}\right) \leq Q_{1}\left(\left\|A_{\sigma}\right\|_{H_{0}}\right) e^{-k_{0}^{\prime}(t-\tau)},
$$

for two appropriate constants $C$ and $k_{0}^{\prime \prime}$.
Since $\sigma=\min \left\{\frac{1}{4}, \frac{5-\gamma}{2}\right\}$ is fixed, by finite steps, we can infer that there is a bounded (not only in $H_{0}$, but also $H_{1}$ ) set $B_{1} \subset H_{1}$ such that

$$
\operatorname{dist}_{H_{0}}\left(U_{g}(t, \tau) B_{0}, B_{1}\right) \leq Q\left(\left\|B_{0}\right\|_{H_{0}}\right) e^{-v(t-\tau)}
$$

Note further that all the constants in (62) only depend on $\left\|B_{0}\right\|_{H_{0}}$ and $\|g\|_{L_{b}^{2}}$.

Now, for any bounded (in $H_{0}$ ) B , from Theorem 3.2 There is $T_{0} \geq \tau$ such that

$$
\bigcup_{\sigma \in \Sigma} U_{g}(t, \tau) B \subset B_{0} \text { for all } t \geq T_{0}
$$

Combined with Lemma 3.4, it follows that

$$
\operatorname{dist}_{H_{0}}\left(U_{g}(t, \tau) B, B_{0}\right) \leq Q e^{v T_{0}} e^{-v(t-\tau)},
$$

where $Q=\sup \left\{\left\|U_{g}(t, \tau) B\right\|_{H_{0}}: g \in \sum, \tau \leq t \leq T_{0}\right\}<\infty$.

Finally, we apply Lemma 2.1, again to (62) and (63), and the proof of Theorem is completed.

\section{UNIFORMLY ATTRACTORS}

Now collecting Theorem 3.2, Lemma 3.5, and Theorem 3.12, we establish that $\left\{U_{g}(t, \tau)\right\}, g \in \sum$ corresponding to (1) is asymptotically compactness. Therefore, by means of well-known results of the theory of dynamical systems we get that the family of processes $\left\{U_{g}(t, \tau)\right\}, g \in \sum$ corresponding to (1), posses a compact (in $H_{0}$ ) uniform (w.r.t $g \in \sum$ ) attractor $\mathcal{A}$, and $\mathcal{A} \subset H_{0}$. We remark that the above existence does not require any continuity of the family of processes. However, in order to obtain the explicit form of $\mathcal{A}$, we need some continuity. Moreover, since the symbol space $\sum$ now has only weak compactness, we need to verify the corresponding of weak continuity. First, by the results of Chepyzhov and Vishik[6], we see that $\sum$ with the local weak convergence topology of $L_{\text {loc }}^{2}\left(R ; L^{2}(\Omega)\right)$ forms a sequentially compact and metrizable complete space. We denote the equivalent metric by $d($,$) .$ Thus $\left(\sum, d\right)$ is a compact metric space. Moreover, through Lemma 4.1, Chapter V[6], we also have the following conclusion.

Lemma 4.1. [13] The translation semigroup $\{T(t)\}_{t \geq 0}$ acts on $\sum$ (i.e.), $T(t) g(x, s)=g(x, t+s)$ for any $g \in \sum$ and any $t \geq 0$ is invariant and continuous in $\sum$ with respect to the local weak convergence topology of $L_{l o c}^{2}\left(R ; L^{2}(\Omega)\right)$, equivalently, with respect to the metric $d$.

In the following, we also recall an useful lemma, whose proof is simple and we omit it. 
Lemma 4.2. [13] Let $X$ be a reflexive Banach and $x_{n} \stackrel{\text { 弱 }}{\longrightarrow} 0$ in $X$. then for each compact (in $X^{*}$ ) subset $B \subset X^{*}$, the uniform convergence hold: For any $\varepsilon>0$ there is a $N_{\varepsilon}$, depending only on $\varepsilon$, such that

$$
\left|<f, x_{n}>_{X^{*}}\right| \leq \varepsilon \text { for all } n \geq N_{\varepsilon} \text { and all } f \in B
$$

Theorem 4.3. The family of processes $\left\{U_{\sigma}(t, \tau)\right\}, \sigma \in \sum$, corresponding to (1.1), has a compact uniform (w.r.t. $\sigma \in \sum$ ) attractor $\mathfrak{I}$ in $H_{0}$. Moreover, this attractor is bounded in $H_{1}$ and can be decomposed as follows

$$
\mathfrak{I}=\bigcup_{\sigma \in \Sigma} \kappa_{\sigma}(0)
$$

where $\kappa_{\sigma}$ is the kernel of the process $\cup_{\sigma}$, and $\kappa_{\sigma}(0)$ is the kernel section at time 0 .

Proof We only need to verify continuity claim on the attractor $\mathfrak{I}$ in $H_{0}$, i.e., for the attractor $\mathfrak{I}$ in $H_{0}, \mathfrak{I} \subset H_{0}$ any fixed $\tau \in R$ and $t \geq \tau$, if $z_{n \tau} \rightarrow z_{\tau}$ in $\mathfrak{I}$ and $g_{n} \rightarrow g$ with respect to the local weak convergence topology of $L_{l o c}^{2}\left(R ; L^{2}(\Omega)\right)$, then $U_{g_{n}}(t, \tau) Z_{n \tau}$ converges to $U_{g}(t, \tau) z_{\tau}$ in I .

\section{Denoted}

$$
z_{\tau}=z_{1 \tau}-z_{2 \tau},\left(u_{i}(\mathrm{t}), u_{i t}(\mathrm{t}), \eta^{t}\right)=\mathrm{U}_{g_{i}}(t, \tau) z_{i \tau}(i=1,2)
$$
$\left(w(\mathrm{t}), w_{t}(\mathrm{t}), \zeta^{t}\right)=\mathrm{U}_{g 1}(t, \tau) z_{1 \tau}-\mathrm{U}_{g 2}(t, \tau) z_{2 \tau}, z_{i \tau} \in \mathfrak{J}, i=1,2$. Then $\left(w(t), w_{t}(t), \zeta^{t}\right)$ satisfies the following equation

$$
w_{t t}-\Delta w-\Delta w_{t}-\Delta w_{t t}-\int_{0}^{\infty} \mu(s) \Delta \zeta^{\zeta}(s) d s+f\left(u_{1}\right)-f\left(u_{2}\right)=g_{1}(x, t)-g_{2}(x, t),
$$

and

$$
\zeta^{t}(s)=w(t)-w(t-s), \quad\left(w(\tau), w_{t}(\tau), \zeta^{\tau}\right)=z_{\tau},\left.\quad w\right|_{\partial \Omega}=0 .
$$

Since $\mathfrak{I}$ is bound in $H_{1}$, following Theorem 3.12, then there is a positive constant $R_{0}$ such that

$$
\sup _{g \in \Sigma} \sup _{\tau \in R} \sup _{t \geq \tau}\left\|\bigcup_{g}(t, \tau) \Im\right\|_{H_{1}}^{2} \leq R_{0}<\infty
$$

Multiplying (66) by $w_{t}(t)$ and using (67), it follows that

$$
\begin{aligned}
& \frac{d}{d t}\left(\left|w_{t}(t)\right|_{2}^{2}+\|w(t)\|_{0}^{2}+\left\|w_{t}(t)\right\|_{0}^{2}+\left\|\zeta^{t}\right\|_{\mu, \varepsilon_{0}}^{2}\right)+2\left\|w_{t}(t)\right\|_{0}^{2}+\delta\left\|\zeta^{t}\right\|_{\mu, \varepsilon_{0}}^{2} \\
& \leq C\left(\left|w_{t}(t)\right|_{2}^{2}+\|w(t)\|_{0}^{2}+\left\|w_{t}(t)\right\|_{0}^{2}+\left\|\zeta^{t}\right\|_{\mu, \varepsilon_{0}}^{2}\right)+2<g_{1}(t)-g_{2}(t), w_{t}(t)>
\end{aligned}
$$

and by integrating over $[\tau, t]$, then we get, for each $\tau \leq t \leq T$,

$$
\begin{aligned}
& \left|w_{t}(t)\right|_{2}^{2}+\|w(t)\|_{0}^{2}+\left\|w_{t}(t)\right\|_{0}^{2}+\left\|\zeta^{t}\right\|_{\mu, \varepsilon_{0}}^{2} \\
& \leq e^{C(T-\tau)}\left(\left\|z_{\tau}\right\|_{H_{0}}^{2}+\left|\int_{\tau}^{T}<g_{1}(s)-g_{2}(s), w_{t}(s)>d s\right|\right) .
\end{aligned}
$$

By Theorem 3.2, then we have

$$
\bigcup_{g \in \Sigma}\left\{\prod_{2} \underset{g}{\bigcup}(t, \tau) z_{\tau}: t \in[\tau, T], z_{\tau} \in \mathfrak{I}\right\} \text { is bounded in } L^{2}\left(\tau, T ; H_{0}^{1}(\Omega)\right)
$$

and

$\bigcup_{g \in \Sigma}\left\{\partial_{t} \prod_{2} \underset{g}{U}(t, \tau) Z_{\tau}: t \in[\tau, T], z_{\tau} \in \mathfrak{T}\right\}$ is bounded in $L^{2}\left(\tau, T ; H_{0}^{1}(\Omega)\right)$ then

$\bigcup_{g \in \Sigma}\left\{\partial_{t} \prod_{2} \underset{g}{U}(t, \tau) z_{\tau}: t \in[\tau, T], z_{\tau} \in \mathfrak{T}\right\}$ is bounded in $L^{2}\left(\tau, T ; H^{-1}(\Omega)\right)$

where $\Pi_{2}$ is the projector from $X \times Y$ to $Y$. Then by Lemma 2.2, we get

$\bigcup_{g \in \Sigma}\left\{\prod_{2} \underset{g}{U}(t, \tau) z_{\tau}: t \in[\tau, T], z_{\tau} \in \mathfrak{I}\right\}$ is compact in $L^{2}\left(\tau, T ; L^{2}(\Omega)\right)$

By Lemma 4.2, it does show that if $g_{n} \rightarrow g$ in $L_{w, l o c}^{2}\left(R ; L^{2}(\Omega)\right)$, then

$$
\left|\int_{\tau}^{t}<g_{1}(s)-g_{2}(s), w_{t}(s)>d s\right| \rightarrow 0
$$

uniformly on a compact subset of $L^{2}\left(\tau, t ; L^{2}(\Omega)\right)$.

Based on the continuity claim above, and by constructing a skew-product flow on $\mathfrak{I} \times \sum$ and applying Theorem 5.1,IV[6], then the structure equality (65)is proved. So the proof is completed.

\section{ACKNOWLEDGMENT}

This work was supported by National Natural Science Foundation of China(Nos71471020) and Hunan Provincial Innovation Foundation for Postgraduate (No.CX2017B491).

\section{REFERENCES}

[1] L. Bogolubsky, Some examples of inelastic soliton interaction, Computer Physics Communications 13(1977): 149-155.

[2] J. Arrieta, A. N. Carvalho and J. K. Hale, A damped hyperbolic equations with critical exponents, Comm. Partial Differential Equations, 17(1992): 841-866.

[3] C. E. Seyler and D. L. Fanstermacher, A symmetric regularized long wave equation, Phys. Fluids 27(1)(1984): 58-66.

[4] M. Conti, E. M. Marchini, V. Pata, A well posedness result for nonlinear viscoelastic equation with memory. Nonlinear Appl-TMA. 94(2014): 206-216.

[5] R. O. Araujo, T. F. Ma, Y. Qin, Long-time behavior of a quasilinear viscoelastic equation with past history, J. Diff. Eqs. 254(10)( 2013): 4066-4087. 
[6] V.V. Chepyzhov, M.I. Vishik, Attractors for equations of mathematical physics, Amer. Math. Soc. Colloq. Publ., Vol. 49, Amer. Math. Soc., Providence, RI, 2002.

[7] M. Grasselli, V. Pata, Asymptotic behavior of a parabolic-hyperbolic system. Commun. Pure Appl.Anal.,3(4)(2004): 849-881.

[8] Y. Qin, B. Feng, M. Zhang, Uniform attractors for a non-autonomous viscoelastic equation with a past history, Nonlinear Anal-TMA. 101(2014):1-15.

[9] H. YassineA. Abbas, Long-time stabilization of solutions to a nonautonomous semilinear viscoelastic equation, Appl. Math. Optim., 73(2016): 251-269.

[10] Y. Xie, C. Zhong, Asymptotic behavior of a class nonlinear evolution equation, Nonlinear Anal-TMA. 71 (2009): 5095-5105.

[11] V. Pata and S. Zelik, Smooth attractors for strongly damped wave equations, Non linearity, 19(2006), 1495-1506.

[12] J. C. Robinson, Infinite-dimensional dynamical dystems, Cambridge University Press,2001.

[13] C. Sun, D. Dao, J. Duan, Non-autonomous wave dynamics with memory-asymptotic regularity and uniform attractor, Disc. Cont. Dyna. Syst., 9(3)(2008): 743-761.

[14] C. Sun and M. Yang, Dynamics of the nonclassical diffusion equations, Asympt. Anal. 59 (2008): 51-81.

[15] P. Fabrie, C. Galushinski, A. Miranville and S. Zelik, Uniform exponential attractors for a singular perturbed damped wave equation, Disc. Cont. Dyn. Sys., 10(1-2)(2004): 211-238.

[16] Y. Xie, Q. Li and K. Zhu, Attractors for nonclassical diffusion equations with arbitrary polynomial growth nonlinearity, Nonlinear Anal-RWA. 31(2016): 23-37.

[17] Y. Xie, Y. Li and Y. Zeng, Uniform attractors for nonclassical diffusion equations with memory, J. Func. Space, 2016(2016):5340489. 